\title{
Ensayos clinicos en enfermedades infecciosas desatendidas en contexto de la globalización: enfoque ético y de derechos humanos
}

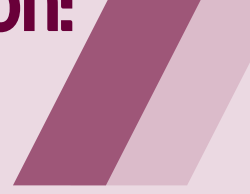

\author{
Clinical trials in neglected \\ infectious diseases in the \\ context of globalization: an \\ ethical and human rights \\ approach
}

\section{RESUMEN}

InTRODUCCIón. Recientes epidemias de enfermedades infecciosas desatendidas (EID) traen a colación problemas existentes como el poco desarrollo de nuevos medicamentos por parte de la industria farmacéutica. Se genera así poca o nula oferta de medicamentos contra dichas enfermedades. Dentro del proceso de globalización, la industria farmacéutica viene desarrollando una estrategia multinacional para nuevos medicamentos según su interés en los paises en vías de desarrollo. Dado que el tema tiene repercusiones en los aspectos económico, ético, político y de derechos humanos, se busca ampliar el conocimiento sobre el proceso de ejecución de ensayos clínicos (EC) sobre EID en Perú. Materiales y método. Se revisaron 1493 EC autorizados en el Perú entre julio de 1995 y junio 2015. Cada uno de los títulos correspondientes a las EID autorizadas fue verificado según el código del proyecto que figura en la página web del Instituto Nacional de Salud. Resultados. En los 20 años evaluados, se autorizaron 17 EC y 2 estaban en proceso de autorización. Los hallazgos representaron $1,3 \%$ del total de los protocolos autorizados; es decir, 0,95 estudios clínicos sobre EID por año de estudio. La razón EID/no-EID fue 1/78. Los EC en EID realizados en Perú correspondieron a cisticercosis, leishmaniosis, estrongiloidiasis, dengue, fiebre amarilla y geohelmintiasis, entre otros parásitos, como criptosporidiosis. Conclusiones. Entre julio 1995 y junio 2015, en Perú hubo un escaso número de ensayos clínicos sobre nuevos medicamentos contra las enfermedades infecciosas desatendidas; esto es responsabilidad de quienes están a cargo de desarrollar nuevos medicamentos. En consecuencia, las personas que requieran dichos medicamentos tendrán poco o nulo acceso a ellos.

Palabras clave: ensayo clínico; enfermedades infecciosas; Perú; ética; investigación; derechos humanos

\section{Hernán A. Sanabria-Rojas}

1 Médico cirujano. Especialista en Enfermedades infecciosas y tropicales. Instituto Nacional de Salud. Oficina General de Investigación y Transferencia Tecnológica.

Departamento de Medicina Preventiva y Salud Pública. Facultad de Medicina de la Universidad Nacional Mayor de San Marcos. Lima, Perú.

\begin{abstract}
IntRoduction. Recent epidemics of neglected infectious diseases (NTD) bring up some existing problems such as underdevelopment of new drugs by the pharmaceutical industry and national states. Little or no supply of medicines against such diseases is thus generated. Within the process of globalization, the pharmaceutical industry has been developing a multinational strategy of developing new drugs according to their interest in developing countries. Since the issue has repercussions in the economic, ethical, political and human rights aspects, it seeks to expand knowledge on the process of implementation of clinical trials (CT) on NTD in Peru. Materials AND METHOD. EC authorized 1493 were revised in Peru between July 1995 and June 2015. Each of the titles to the authorized EID was verified by the project code contained on the website of the National Institute of Health. Results. In the twenty years evaluated, they were authorized 17 CT and two others were in the process of authorization. The findings represented 1.3\% of total authorized protocols; i.e., 0.95 clinical trials on clinical trials per year of study. The EID/no-EID reason was $1 / 78$. The CT on EID performed in Peru accounted for cysticercosis, leishmaniasis, strongyloidiasis, dengue, yellow fever and geoheliminthiasis, among other parasites such as cryptosporidiosis. Conclusions. Between July 1995 and June 2015, in Peru there was a small number of clinical trials on new drugs for neglected infectious diseases; this is the responsibility of those in charge of developing new drugs. Consequent$l y$, people who require these drugs have little or no access to them.
\end{abstract}

KEY wORDs: clinical trial; infectious diseases; Peru; ethics; research; human rights 


\section{INTRODUCCIÓN}

Según Casals, ${ }^{1}$ la integración global en los terrenos social, político, económico y cultural refleja el proceso denominado globalización, el cual emana de dos fuentes: del desarrollo de las nuevas tecnologías de la información y de los procesos de reestructuración en el modo de funcionamiento de la economía del capital. Al respecto, Stiglitz ${ }^{2}$ señala: "La globalización es la aceptación del capitalismo triunfante como única vía posible de progreso, que dificulta la redistribución de la riqueza, concentra el capital, produce pobreza y desempleo y tiene gran impacto sobre la vida, el ambiente y la salud". Para Feo, ${ }^{3}$ el rasgo fundamental de la globalización es la imposición de un modelo de pensamiento y una forma de concebir el mundo, la sociedad, la producción y distribución de bienes y las relaciones entre naciones: el neoliberalismo.

Kanter $^{4}$ habla sobre la cuestión política y los mecanismos de poder, diciendo: "La globalización caracterizaría la pérdida o relajamiento del control político por parte de los gobiernos nacionales, expresado en el abandono progresivo de las funciones reguladoras frente al avance del proceso de centralización de la toma de decisiones económicas; los estados pasarían de hacedores de decisiones (decision makers) a tomadores de decisiones (decision takers)". Esto hace que haya una coordinación cada vez más estrecha con los gigantes industriales, como la industria que produce medicamentos. La globalización como fenómeno multidimensional abarca y se proyecta sobre diferentes campos de actividad: económico, político, tecnológico, militar, jurídico, cultural, medioambiental y de la salud, con formas de interacción, estilos e intensidades diferentes.

Acorde con la OMS, la falta de acceso a los medicamentos necesarios en el mundo es una violación a los derechos humanos y constituye uno de los problemas más importantes de salud pública. ${ }^{5}$ Las Naciones Unidas incluyen el acceso a los medicamentos entre los indicadores para medir el progreso en salud de las naciones. ${ }^{6}$ Potenciar el acceso y uso apropiado de los medicamentos es una forma de disminuir las desigualdades sociales, dado que los pobres gastan un porcentaje más alto de su presupuesto para la salud, en comparación con las clases más pudientes.? Se estima que solo una tercera parte de la población mundial tiene acceso a los medicamentos que necesita; es peor en el caso de las enfermedades infecciosas desatendidas. La globalización influye sobre la salud dado que esta se encuentra estrechamente vinculada con variables sociales y determinada por factores económicos. Al respecto, la OMS viene enfatizando el enfoque de los determinantes sociales de la salud, ${ }^{8}$ además del enfoque biomédico, cuyos resultados no han sido tan halagadores con respecto a la prevención y control de las enfermedades. Sin embargo, los grandes avances en medicina y salud continúan dándose en el área biomédica, en especial en la investigación terapéutica, al probarse nuevos medicamentos con regulaciones estrictas para proteger a los sujetos de investigación y obtener resultados de calidad en beneficio de la humanidad. ${ }^{9}$

El modelo de desarrollo hegemónico actual puede dar lugar a que las enfermedades y las epidemias se agraven por el debilitamiento de los servicios de salud y el recorte de presupuestos internacionales, aunado a la precarización de ciertas zonas del planeta debido a la globalización, lo que crea condiciones sanitarias deprimentes y favorables para el desarrollo de epidemias. En este aspecto, los países en vías de desarrollo son los más afectados, particularmente los situados entre los dos trópicos, el de Cáncer y el de Capricornio, con las denominadas «enfermedades tropicales». El impacto epidemiológico de la globalización se traduce en el mantenimiento, emergencia y reemergencia de enfermedades infecciosas, muchas de ellas enfermedades infecciosas desatendidas (EID). La Organización Mundial de la Salud (OMS) se refiere a las EID como neglected tropical disease (NTD, por sus siglas en inglés).

La industria farmacéutica mundial viene desarrollando una estrategia multinacional para llevar a cabo la investigación clínica y el desarrollo de nuevos medicamentos. ${ }^{10}$ Un ejemplo de EID es la enfermedad del virus del Ébola, que se ha venido propagando en África Occidental, particularmente en Guinea, Liberia, Sierra Leona y Nigeria, desde diciembre de 2013; es considerado el brote epidémico más letal de la enfermedad, según 
información de la OMS,11 la cual, en su reporte del 27 de mayo 2015, comunicó la existencia de más de 25000 casos y 10000 muertes, aparte de cientos de trabajadores de la salud con la enfermedad, en los países mencionados. Han fallecido cerca de $60 \%$ de los infectados, que es una tasa importante de mortalidad; ${ }^{12}$ sin embargo, el Ébola puede provocar la muerte de $90 \%$ de los infectados. ${ }^{13}$ Entre tanto, en África Central existe poca investigación sobre el tratamiento, desde que se dio el primer caso, hace aproximadamente 40 años, hasta la actualidad. En el brote 2013-2015, se ha probado el ZMapp (un suero inmunológico experimental, que contiene tres anticuerpos monoclonales de altísima complejidad), pero que nunca fue usado en humanos antes del brote, pero sí en monos y con resultados variables. ${ }^{11}$ Es tarea de los estados nacionales responsabilizarse por la salud de sus ciudadanos, más aun cuando la industria farmacéutica no produce medicamentos para las enfermedades endémicas.

El desinterés de la industria farmacéutica y de los responsables de la salud pública en los países ha llevado al fracaso de las intervenciones de prevención y control en la gran mayoría de casos de EID. ${ }^{14}$ Se reconoce la existencia de problemas en el grupo de enfermedades tropicales. ${ }^{15}$ Enfermedades hemorrágicas, como las producidas por los virus del Ébola y Marburgo en el África, evidencian que hay otro grupo de enfermedades que, a pesar de reunir casi todas las características de las EID, no se encuentran en el listado de la OMS. ${ }^{16}$ Estas otras EID podrían, incluso, estar más desatendidas y ser más graves. Los infectólogos y tropicalistas peruanos somos testigos de la persistente escasez de terapias para la amplia gama de EID, a pesar del progreso de la investigación científica. Como sucedió con el tratamiento para la enfermedad del virus del Ébola, hay una insuficiente producción de nuevas alternativas preventivas y terapéuticas para las EID, lo que conlleva a la inequidad entre pacientes pobres con relación al resto de población.

En el listado de 17 EID de la OMS ${ }^{16}$ no se incluyen muchas de nuestras enfermedades infecciosas nacionales. Al respecto, la investigación con ensayos clínicos es normado por el Reglamento de ensayos clínicos, aprobado por el Decreto Supremo
N. 017-2006-SA y modificado por el Decreto Supremo N. ${ }^{\circ}$ 006-2007-SA. ${ }^{17}$ Allí se contempla que la autoridad reguladora es el Instituto Nacional de Salud.

En este sentido, el objetivo de este estudio fue ampliar el conocimiento sobre el proceso de ejecución de los ensayos clínicos (EC) sobre EID autorizados por el Instituto Nacional de Salud en los últimos 20 años de globalización.

\section{MATERIALES Y MÉTODO}

Se hizo la revisión de todos los EC autorizados por el Instituto Nacional de Salud (INS) en el Perú en los últimos 20 años, desde julio de 1995 hasta junio de 2015. Se encontró un total de 1493 EC autorizados. Se revisó la base de datos publicada en la página web del INS. Cada uno de los títulos de los EID autorizados fueron verificados según el código del proyecto que se encuentran en el actual Registro Peruano de Ensayos Clínicos (Repec).

Como criterio de inclusión se consideró: ensayo clínico registrado en las áreas de Infectología y/o Medicina Tropical/Parasitología, en el Repec y que el tema tratado cumpla con la definición de enfermedades infecciosas desatendidas, dada por la OMS. ${ }^{16}$ De este modo, se consideraron las siguientes enfermedades: dengue y chikunguña o chikungunya, rabia, tripanosomiasis sudamericana y africana, leishmaniosis, úlcera de Buruli, lepra, tracoma, pian, cisticercosis y teniasis, dracunculiasis, fascioliasis, hidatidosis y geohelmintiasis. Asimismo, las EID propuestas por Cabezas $^{15}$ para el Perú: enfermedad de Carrión, peste, leptospirosis, brucelosis, HTLV I-II, micosis subcutánea como la esporotricosis, las micosis profundas (como la paracoccidioidomicosis), la fiebre amarilla, fiebres hemorrágicas como la enfermedad por hantavirus, la fiebre de Mayaro, fiebre de oropouche y la encefalitis equina venezolana; finalmente, los envenenamientos por ofidismo, loxocelismo y lonomiasis. También se considera EID a la filariasis por Mansonella ozzardi, encontrada en Iquitos por Vargas et al. ${ }^{18}$ La esporotricosis y la paracoccidioidomicosis ya habían sido propuestas por Zurita ${ }^{19}$ como EID. Finalmente, se incluyó, ex profeso, a la criptosporidiosis. 


\section{RESULTADOS}

Se identificaron 1178 EC autorizados por el INS en los 20 años de estudio, correspondientes a la especialidad de Infectología y/o Medicina Tropical/Parasitología, catalogadas según la definición operacional de «enfermedades infecciosas desatendida», para el presente estudio. En estos 20 años, solo se autorizaron 17 EC y otros dos más aún estaban en proceso de autorización; en total, 19 EC, que representan el $1,3 \%$ del total de los protocolos autorizados; es decir, un 0,95 estudios clínicos sobre EID por año de estudio. La razón EID/no-EID fue 1/78. Los EC sobre EID realizados en el Perú correspondieron a: cisticercosis, leishmaniosis, estrongiloidiasis, dengue, fiebre amarilla y geohelimintiasis, entre otros parásitos, como la criptosporidiosis.

La mayoría de las investigaciones fueron patrocinadas por la industria farmacéutica y, en menor frecuencia, por la universidad peruana, particularmente la Universidad Peruana Cayetano Heredia. Solamente dos ensayos clínicos fueron patrocinados por el Estado Peruano a través del INS, sobre medicamentos contra la malaria. El primer estudio fue realizado en el año 2004, Eficacia de tres esquemas diferentes de primaquina para la prevención de recaídas de malaria por Plasmodium vivax en la región amazónica del Perú; el otro ensayo fue autorizado en 2013, Evaluación de la eficacia in vivo e in vitro de la terapia combinada artesunato/mefloquina para el tratamiento de la malaria por Plasmodium falciparum no complicada en la Amazonía peruana. Ambos estudios se realizaron con el coauspicio del Instituto de Investigación de Enfermedades Tropicales, de la Marina de los EE. UU. (Namru-6), con sede en Lima, Perú.

\section{DISCUSIÓN}

Como se sabe, la industria viene desarrollando una estrategia multinacional para llevar a cabo la investigación clínica y desarrollo de nuevos medicamentos, ${ }^{10}$ consistente en realizar protocolos de investigación en una muestra grande de pacientes enfermos en múltiples países simultáneamente $y$, en particular, en países menos desarrollados, donde pueden obtener pacientes e instalaciones con mayor facilidad que en los países de origen de los diseños, como los Estados Unidos, Japón y algunos países de la Unión Europea, entre otros.

En Perú, el número de investigaciones realizadas sobre las EID es pequeño, según se ha demostrado con los registros del INS, institución responsable de las autorizaciones en el país. Llama la atención, por un lado, que solo hayamos encontrado en promedio un EC de EID por cada año, en los últimos veinte años, pese a que se incluyeron algunas otras enfermedades tropicales, además de las definiciones de la OMS y la lista propuesta para el Perú por Cabezas. ${ }^{15}$

Pero, por otro lado, no sorprenden los resultados, puesto que, según se conoce, la industria farmacéutica invierte poco en el estudio de las EID, que en su mayoría se manifiestan en países en vías de desarrollo y entre la población pobre. Al respecto, un estudio publicado en la revista Lancet encontró que de las 1556 nuevas drogas desarrolladas y registradas por las industrias farmacéuticas entre 1974 y 2004, solo diez estaban destinadas a enfermedades exclusivas de países en vías de desarrollo. ${ }^{20}$ En esas tres décadas hubo la expansión de los EC hacia los países "periféricos", pero esta expansión no puede ser considerada realmente "benéfica" para los países en vías de desarrollo, como en América Latina. ${ }^{21}$ Otro estudio señala que solo $4 \%$ de los nuevos fármacos y vacunas aprobados entre 2000 y 2011 en todo el planeta fue para tratar las llamadas enfermedades olvidadas. ${ }^{22}$

Un estudio de Minaya et al., ${ }^{23}$ realizado para describir las principales características de los ensayos clínicos (EC) autorizados en Perú desde 1995 hasta agosto de 2012, encontró que $15 \%$ de ensayos correspondieron al área de Infectología y $1,2 \%$ al de las EID; este es un porcentaje muy similar al 1,3\% de nuestros hallazgos. Es deseable que se siga tendencia ascendente en los años venideros; pero para ello, deben confluir diversos factores; mientras esto no suceda, los hallazgos seguirán reflejando una insuficiente producción de nuevas alternativas preventivas y terapéuticas para las EID, lo que contribuirá a una mayor inequidad en salud.

Nuestros hallazgos se contraponen a lo señalado en el numeral «f» de los objetivos de la Declaración 
Universal sobre Bioética y Derechos Humanos,24 dado para los estados: «promover un acceso equitativo a los adelantos de la medicina, la ciencia y la tecnología, así como la más amplia circulación posible y un rápido aprovechamiento compartido de los conocimientos relativos a esos adelantos y de sus correspondientes beneficios, prestando una especial atención a las necesidades de los países en desarrollo».

Nuestros hallazgos sobre los últimos veinte años difieren de lo realizado entre 1910 y 1970, cuando se desarrollaron de medicamentos para combatir las EID: tripanomicidas y antiamebiásicos, en los años treinta; la cloroquina, en los años cuarenta y cincuenta y los antihelmínticos, en los años sesenta. Sin embargo, la investigación de enfermedades tropicales endémicas dejó de ser fructífera a partir de la década de 1980. Según Vidal,25 las estrategias del mercado cambiaron y hubo menor desarrollo de medicamentos, que llegó a 30,9\%; específicamente, para las enfermedades tropicales llegó a 1\%, similar a lo encontrado en el presente estudio. En este campo no hay progreso, la gente sigue sufriendo y muriendo por las EID y los responsables sanitarios no son capaces de ofrecer a todos estos pacientes el mejor tratamiento posible.

Los hallazgos de la presente investigación no hacen más que corroborar la situación lamentable de los esfuerzos por encontrar tratamientos para las EID. Los estudios clínicos para las EID a nivel mundial son escasos, pues de los casi 150000 EC registrados para el desarrollo de nuevos productos terapéuticos hasta diciembre de 2011 , solo $1 \%$ correspondía a enfermedades desatendidas. ${ }^{22}$ Pero lo más importante es que estos EC son escasos en las zonas geográficas en donde se generan y permanecen estas enfermedades $y$, por lo tanto, sus prevalencias son altas. El problema de que existan pocos medicamentos disponibles para las EID está relacionado con el financiamiento que deben proveer los estados nacionales, pero también tiene implicancias éticas, de derechos humanos y de salud pública. En los estados donde se presentan las EID se podrían generar alianzas con la industria farmacéutica, si no es posible el patrocinio directo, con la finalidad de promover el desarrollo de moléculas químicas para combatir dichas enfermedades. De lograrse, lo que sin duda es dificultoso, abriría el camino para encontrar estrategias que permitan disminuir la incidencia y prevalencia de las EID en el mundo. Mientras tanto, da la impresión que la industria farmacéutica y los gobiernos se preocupan más por la salud de los que pueden pagar los medicamentos.

Si la salud es un derecho universal, reconocido jurídicamente por las principales instituciones internacionales, entonces no se está cumpliendo la Declaración Universal de los Derechos del Hombre, pues su artículo 25 dice: "Toda persona tiene derecho a un nivel de vida adecuado que le asegure, así como a su familia, la salud, el bienestar $y$, en especial, la alimentación, el vestido, la vivienda, la asistencia médica y los servicios sociales necesarios". 26 "El mejor estado de salud que se pueda obtener constituye uno de los derechos fundamentales de todo ser humano, sea cual sea su raza, religión, opiniones políticas, condición económica o social", reza la carta de constitución de la OMS, organismo de la ONU. En otras palabras, la salud es un derecho al que todo individuo puede acceder sin diferencias de ningún tipo; es universal, para todos los seres humanos. Si observamos la cantidad de cartas, acuerdos, declaraciones, etc. que hacen referencia a la igualdad entre hombres y mujeres, adultos y niños, blancos y negros y personas con capacidades diferentes, vemos que en el Perú actual no estamos muy alejados de lo que los españoles se plantearon en la época de la conquista.

Atender a los ciudadanos peruanos que sufren de EID es tarea del Estado Peruano y del gobierno correspondiente, particularmente cuando se evidencia que la industria farmacéutica no ha producido suficientes medicamentos para dichas enfermedades.

\section{CONCLUSIONES}

En el período de julio de 1995 a junio de 2015, en Perú hubo un escaso número de ensayos clínicos sobre nuevos medicamentos contra las enfermedades infecciosas desatendidas; esto es responsabilidad de quienes están a cargo de desarrollar nuevos medicamentos. En consecuencia, las personas que requieran dichos medicamentos tendrán poco o nulo acceso a ellos. 


\section{RECOMENDACIONES}

Al haber escasos EC y, consiguientemente, menor producción de medicamentos para los ciudadanos peruanos que necesitan tratamiento de EID, el Estado Peruano debiera responsabilizarse de ejecutar los EC necesarios para esos ciudadanos, al no ser de interés de la industria farmacéutica las EID.

\section{REFERENCIAS BIBLIOGRÁFICAS}

I. Casals, C. Globalización: apuntes de un proceso que está transformando nuestras vidas. Barcelona, España: Intermón; 2004.

2. Stiglitz JE. El rumbo de las reformas: hacia una nueva agenda para América Latina [Internet]. CEPAL. 2003;(80):7-40 [citado 10 ago 2014]. Disponible en: http://repositorio.cepal.org/bitstream/ handle/ I 1362/10893/080007040_es.pdf?sequence= I

3. Feo O. Reflexiones sobre la globalización y su impacto sobre la salud de los trabajadores y el ambiente. Ciênc Saúde Coletiva. 2003;8(4):887-96.

4. Kanter RM, Pittinsky TL. Globalization: new worlds for social inquiry. Berkeley J Sociology. 1996;40: I-20.

5. Hogerzeil HV, Mirza Z.The world medicines situation 20I I:access to essential medicines as part of the right to health [Internet]. Ginebra, Suiza:WHO; 2011 [citado 10 ago 2014]. Disponible en: http://apps.who.int/medicinedocs/documents/s 18772en/s 18772en.pdf

6. United Nations, Office of the High Commissioner for Human Rights. Report on indicators for promoting and monitoring the implementation of human rights. Document HRI/MC/2008/3 [Internet]. Ginebra, Suiza: United Nations, OHCHR; 2008 [citado 10 ago 2014]. Disponible en: http://www2.ohchr.org/english/ issues/indicators/docs/HRI.MC.2008.3_en.pdf

7. Makinen $M$, Waters $H$, Rauch $M$, Almagambetova $N$, Bitran $R$, Gilson $\mathrm{L}$, et al. Inequalities in health care use and expenditures: empirical data from eight developing countries and countries in transition. Bull World Health Organ. 2000;78(I):55-65.

8. Organización Mundial de la Salud (OMS). Reducir las inequidades sanitarias actuando sobre los determinantes sociales de la salud (Resolución WHA 62.14). Actas de la $62^{\text {a }}$ Asamblea Mundial de la Salud; 22 may 2009 [Internet]. Ginebra, Suiza: OMS; 2009 [citado 10 ago 2014]. Disponible en: http://apps.who.int/gb/ebwha/pdf_ files/A62/A62_RI4-sp.pdf

9. Emanuel E. ¿Qué hace que la investigación clínica sea ética? Siete requisitos éticos. En: Pellegrini A, Macklin R, editores. Investigación en sujetos humanos: experiencia internacional. Santiago de Chile, Chile: OMS; 1999. p. 33-46.

10. Getz KA. Global clinical trials activity in the details [Internet]. Iselin (Nueva Jersey), EE. UU.:Applied Clinical Trials; 2007 [citado 3 ene 2013]. Disponible en: http://www.appliedclinicaltrialsonline. com/contact-applied-clinical-trials

II. Organización Mundial de la Salud (OMS). Declaración de la OMS sobre la reunión del Comité de Emergencias del Reglamento Sanitario Internacional acerca del brote de enfermedad por el virus del Ebola de 2014 en África Occidental; 8 ago 2014 [Internet]. Ginebra:OMS;2014 [citado 10 oct 2014].Disponible en:http://www. who.int/mediacentre/news/statements/2014/ebola-20140808/es/

12. Pruebas anecdóticas sobre los tratamientos experimentales del ébola (Evaluación de la situación); 2 I ago 2014 [Internet]. Ginebra, Suiza: OMS; 2014 [citado 10 oct 2014]. Disponible en: http://www. who.int/mediacentre/news/ebola/2I-august-2014/es/

13. Feldmann H, Geisbert TW. Ebola haemorrhagic fever. Lancet. $2011 ; 377(9768): 849-62$.
14. Trouiller P, Olliaro P, Torreele E, Orbinski J, Laing R, Ford N. Drug development for neglected diseases: a deficient market and a public-health policy failure. Lancet. 2002;359(9324):2188-94.

15. Cabezas-Sánchez C. Enfermedades infecciosas desatendidas: un permanente reto para la salud pública y la equidad en el Perú. Rev Peru Med Exp Salud Publica. 20I4; 3I (2):326-35.

16. World Health Organization (WHO). Neglected tropical diseases: a statistical update - latest data available [Internet]. Ginebra, Suiza: WHO; 2014 [citado 10 oct 2014]. Disponible en: http://www.who. int/neglected_diseases/NTD__A_statistical_update_latest_data_ available.pdf?ua=I

17. República del Perú, Ministerio de Salud. Decreto Supremo № 0 I72006-SA, Reglamento de ensayos clínicos del Perú (promulgado el 29 de julio de 2006); modificado por el Decreto Supremo $\mathrm{N}^{\circ}$ 006-2007-SA (promulgado el 7 de junio de 2007). Lima, Perú: El Peruano, Normas Legales; 2006, 2007 [citado 10 oct 2014]. Disponible en: http://www.ins.gob.pe/insvirtual/images/otrpubs/ pdf/ENSAYOS\%20CLINICOS\%203\%20DE\%20AGOSTO\%20 DE\%2020II.pdf

18. Vargas-Herrera J, Arróspide-Velasco N, Gutierrez-González S, Celis-Salinas JC, Huamani-Solano D, Loza-Hermenegildo L, et al. Reporte de cuatro casos clínicos de filariasis en Alto Nanay, Loreto. Rev Peru Med Exp Salud Publica. 2013;30(3):506-I I.

19. Zurita S. Esporotricosis y paracoccidioidomicosis en Perú: experiencias en prevención y control. Rev Peru Med Exp Salud Publica. 20I4;3I (2):352-7.

20. Chirac P, Torreele E. Global framework on essential health R\&D. Lancet. 2006;367(9522):I560-I.

21. Homedes $N$, Ugalde A. La globalización de la investigación clínica en América Latina. En: Homedes N, Ugalde A, editores. Ética y ensayos clínicos en América Latina. Buenos Aires: Lugar; 2013.

22. Pedrique B, Strub-Wourgaft N, Some C, Olliaro P, Trouiller P, Ford $\mathrm{N}$, et al. The drug and vaccine landscape for neglected diseases (2000-I I): a systematic assessment. Lancet Glob Health. 20I3;।(6):e37।-9.

23. Minaya G, Fuentes D, Obregón C, Ayala-Quintanilla B, Yagui M. Características de los ensayos clínicos autorizados en el Perú, 1995-20 I2. Rev Per Med Exp Salud Publica. 20 12;29(4):43I-6.

24. Organización de las Naciones Unidas para la Educación, la Ciencia y la Cultura (Unesco). Declaración universal sobre bioética y derechos humanos (Resolución $33 \mathrm{C} / 36$ ). Actas de la $33^{\mathrm{a}}$ reunión de la Conferencia General; 19 oct 2005 [Internet]. París, Francia: UNESCO; 2005 [citado 10 oct 2014]. Disponible en: http:// unesdoc.unesco.org/images $/ 00$ | 4/00 I 428//42825s.pdf\#page=85

25. Vidal SM. ¿Ética o mercado?, una decisión urgente: lineamientos para el diseño de normas éticas en investigación biomédica en América Latina. En: Keyeux G, Penschaszadeh V, Saada A, editores. Ética de la investigación en seres humanos y políticas de salud pública. Bogotá, Colombia: Redbioética Unesco, Universidad Nacional de Colombia; 2006. p. 191-232.

26. Organización de las Naciones Unidas (ONU). Declaración Universal de los Derechos del Hombre [Internet]. Ginebra, Suiza: ONU; 1948 [citado 10 ago 2014]. Disponible en: http://www. un.org/es/documents/udhr/index_print.shtml

Correspondencia: Dr. Hernán Sanabria-Rojas hsanabria@ins.gob.pe hernan_@hotmail.com

\section{Conflictos de interés}

El autor declara no tener conflictos de interés durante el planteamiento, ejecución de la investigación y la elaboración del artículo para su publicación.

Fecha de recepción: 3 de septiembre de 2015

Fecha de aceptación: 21 de septiembre de 2015 